\title{
Response to Van Rompaey Vincent to his accepted article: response to: Prognostic indicators of hearing after complete resection of cholesteatoma causing a labyrinthine fistula by Stephenson MF and Saliba I
}

\author{
Issam Saliba $\cdot$ Marie-France Stephenson
}

Received: 9 August 2011/Accepted: 23 August 2011/Published online: 1 September 2011

(C) Springer-Verlag 2011

Thank you for your interest in our study and also for presenting your concerns. Our previous article [1] describing the prognostic indicators of hearing after complete resection of cholesteatoma causing a labyrinthine fistula reported that the preoperative high-resolution thin sliced $0.55 \mathrm{~mm}$ CT-scan is a very precise tool for diagnosing labyrinthine fistulas with a sensitivity and specificity of $100 \%$.

It is well known that the absence of fluid in the semicircular canal (no signal on T2 MRI) or the presence of fluid in the semicircular canal (high-signal on T2 MRI) can help us to identify the presence or not of fibrous tissue in the labyrinth. However, nowadays it is impossible for the MRI to identify the disruption of the membranous labyrinth by the cholesteatoma. The membranous labyrinth could be invaded by the cholesteatoma with no fibrous development in the labyrinth; in these cases of membranous fistula, the risk of postoperative sensorineural hearing loss is higher than the cases of bone fistulas. Once again, in the cases of cholesteatoma, the MRI cannot differentiate the membranous fistula from a bone fistula in the absence of labyrinthine fibrosis. Therefore, it cannot predict the postoperative hearing outcome after a complete removal of the matrix.

In our study, we reported the importance of the highresolution thin sliced CT-scan to identify labyrinthine fistula reaching $3.55 \mathrm{~mm}$ to suspect a membranous fistula with a sensitivity of $66 \%$ and a specificity of $71 \%$. Thus, the preoperative counseling of the patient is more precise and more realistic especially about the postoperative sensorineural hearing loss.

Conflict of interest The author has nothing to declare.

\section{Reference}

1. Stephenson MF, Saliba I (2011) Prognostic indicators of hearing after complete resection of cholesteatoma causing a labyrinthine fistula. Eur Arch Otorhinolaryngol [Epub ahead of print]
I. Saliba $(\bowtie) \cdot$ M.-F. Stephenson

Department of Otolaryngology, Head and Neck Surgery,

Montreal University Hospital Center (CHUM),

Montreal University, 1560 Sherbrooke East, Montreal,

QC H2L 4M1, Canada

e-mail: issam.saliba@umontreal.ca 